Pobrane z czasopisma New Horizons in English Studies http://newhorizons.umcs.pl Data: 26/04/2023 11:42:09

New Horizons in English Studies 6/2021

REVIEW

$\infty$

\title{
Zuzana Kozáčiková
}

Constantine the Philosopher University in Nitra, Slovakia ZKOZACIKOVA@UKF.SK

HTTPS://ORCID.ORG/OOOO-0003-3482-238X

\section{A Few Comments on Disciplinary Differences in Academic Publishing}

\section{Ken Hyland and Feng K. Jiang. Academic Discourse and Global Publishing. Disciplinary Persuasion in Changing Times. New York: Routledge, 2019, pp. 261.}

Disciplinary persuasion in changing times obviously focuses, as the title suggests, on how academic discourse changed over the past 50 years and is therefore viewed as essential reading on academic discourse for both novice and experienced researchers. Professor Hyland has been continuously working on academic discourse in most of his works $(1998,2004,2009)$ and his corpus-based approach is viewed as an immense and valuable source of this in-depth study of disciplinary variations in academic discourse. With four main parts and thirteen chapters, this interesting piece of writing published in 2019 explores the changes in argument patterns, stance and engagement in academic writing and therefore can be viewed as a multifaceted work on academic discourse from the perspective of global publishing.

The concise first part with two main sub-chapters analyses the major reasons for a massive increase in academic publishing. In a very accessible way these reasons are clarified and the main academic, cultural and educational practices in academic discourse are highlighted. Here Professor Hyland clearly explains a number of motives for the rise in academic publishing and analyses four major factors which contribute to this massive explosion of academic writing. These are mainly technological changes, the emergence of open access journals, the increase in the importance of research and 
a growing pressure to publish. What makes this first part really interesting for readers is how accessibly it refers to the changing patterns of academic life and how it answers the question- why is there this explosion in academic writing?The authors defined a number of reasons for this evident growth in academic publishing, admitting that this enormous increase may have a negative impact on other important aspects of academic duties such as editing, reviewing and teaching.

The second part with the title Changes in argument patterns presents, in the authors' words, how research writing has changed in terms of the main communicative functions expressed by writers targeting cohesion and coherence, changing patterns of citation and changes in multi-word combinations. Academic citation plays a crucial role in the scientific discourse community, since it helps to link scientific authors'claims, suggestions and oucomes with wider argumentative schemata. The diachronic changes reflect the major transformations in academic discourse citation such as a relative decline in the use of reporting verbs or the increase in self-citation in sociology, applied linguistics and electrical engineering. It is worth observing some other interesting findings the authors present in the second chapter of their work as "a shift to parenthetical citations, with only a bracketed or superscript reference to the authors" (Hyland and Jiang 2019,105) or "a change in authors' use of reporting verbs in sentential self-citations with a steady shift towards research acts over the past fifty years" (Hyland and Jiang 2019, 103). Noticeably, Hyland and Jiang are pioneers in this field, since there has been no systematic research on how citation practices have changed across time in particular disciplines. In the authors' words, they "investigate a range of discoursal features, some of which have received less attention in the literature than others to determine what has changed and to give some tentative answers to the question of why they have changed" (Hyland and Jiang 2019, xii).

The concept of stance and engagement in academic discourse has already been discussed by many authors (Palmer, Hunston\&Thompson, Quirk) but in Hyland's view stance expressions are disciplinary practices as much as individual variations which reflect authors' own positions in their own writings. Despite the interest in the notion of stance, it is quite evident that very little is known of how stance has changed over time. A very detailed quantitative analysis of stance features targeting the main components of stance (evidentiality, affect and presence) proposed in the third chapter Changes in stance and engagement (p.127-223) shows quite surprising results. By assessing changes in stance over time through four disciplines - applied linguistics, sociology, electrical engineering and biology, Hyland and Jiang came to the conclusion that authors in the soft knowledge fields have exhibited a movement towards considerably less marking of evidentiality and there is a substantial fall in self-mention over the last 50 years. Additionaly, the use of evaluative that as a stance marker has slightly declined but still remains one of the main grammatical devices for marking attitudinal meaning in context. Apart from the above mentioned changes in stance and engagement, the chapter ends with a concept of informality in academic writing. As the authors suggest, informality in academic writing is "the expression of a more personal tenor and this implies a closer 
relationship to readers, a willingness to negotiate claims and a positive attitude towards subjectivity" (Hyland and Jiang 2019, 209). Even though there is a slow tendency for academic writing to become more informal, disciplinary differences are again quite evident with the science and engineering disciplines viewed as less formal.

In Part 4 of the book entitled Epilogue the analyses and changes presented in the book are summarised and reconsidered. In the authors' words in this final chapter they review the analyses presented in the book, offer an explanation for the changes they have found and explore some of the challenges and options they offer researchers, teachers and students (Hyland and Jiang 2019, 227). Based on their findings, it is quite evident that hard knowledge disciplines (e.g. biology and electrical engineering) show apparent preference for some grammatical, lexical and semantic devices which are not used to the same or approximate extent in soft science disciplines (e.g. sociology or applied linguistics). The use of self-citation, for example, is more evident in hard sciences, whereas less visible stance is preferable in soft sciences.

Besides the above-mentioned topics, this book has some other special features which will be welcomed by all its readers such as a very detailed List of references and Index. Additionally, all the tables in the book (listed in List of Tables) are used to organize data that is too detailed or complicated to be described adequately in the text, allowing the reader to quickly see the results. All these tables make the text more readable by removing numeric data from the body of the text. What a prospective reader can appreciate about this book is not only a very detailed analysis of changes in academic publishing over a period of time, but also plenty of examples taken from the corpus comprising 360 papers and 2.2 million words. These are primarily used to illustrate the diachronic changes in academic publishing but may serve for educational purposes as an essential resource for analysing professional texts and discourse. In other words, the book under review is unique not only for its detailed quantitative analyses but what makes it accessible and liked by its readers are definitely the commentaries which link the practical analyses with the main theoretical concepts. Studying language changes in Hyland's and Jiang's view is by no means descriptive, but provides an excellent indepth analytical study of various language means in academic writing over the last fifty years. What makes this book an excellent read not only for the academic community are also its comments which describe academic publishing as a huge industry focusing on a strong competition among academics and career demands to publish. In this view, Hyland and Jiang take academic publishing as "not only a means of disseminating and interrogating research claims but a multi-million-dollar industry which influences the academy in fundamental ways" (Hyland and Jiang 2019, 231). The fluidity of this statement is quite evident when reading the book and it is what makes this book an excellent read for everybody interested in academic publishing.

In conclusion, it should be stressed that this book is a very useful and unique addition to the field. While providing a comprehensive review of the main disciplinary practices in academic discourse, the monograph can be seen as an inspiring and challenging study on how academic discourse has changed over the past 50 years. 